\title{
Nanoparticles isolated from blood: a reflection of vesiculability of blood cells during the isolation process
}

This article was published in the following Dove Press journal:

International Journal of Nanomedicine

7 November 2011

Number of times this article has been viewed

\author{
Vid Šuštar' \\ Apolonija Bedina-Zavec ${ }^{1,2}$ \\ Roman Štukelj' \\ Mojca Frank ${ }^{3}$ \\ Goran Bobojevićl \\ Rado Janša ${ }^{4}$ \\ Eva Ogorevc 5 \\ Peter Kruljc ${ }^{6}$ \\ Keriya $\mathrm{Mam}^{7}$ \\ Boštjan Šimunič ${ }^{8}$ \\ Mateja Manček-Keber ${ }^{9}$ \\ Roman Jerala ${ }^{9}$ \\ Blaž Rozman ${ }^{3}$ \\ Peter Veranič ${ }^{10}$ \\ Henry Hägerstrand"I \\ Veronika Kralj-Iglič
}

'Laboratory of Clinical Biophysics, Faculty of Medicine, University of Ljubljana, Ljubljana,

Slovenia; ${ }^{2}$ Laboratory of Biosynthesis and

Biotransformation, National Institute of

Chemistry, Ljubljana, Slovenia; ${ }^{3}$ Departments

of Rheumatology; ${ }^{4}$ Gastroenterology,

Ljubljana University Medical Centre,

Ljubljana, Slovenia; ${ }^{5}$ Laboratory of

Biophysics, Faculty of Electrical Engineering;

${ }^{6}$ Clinics for Reproduction and Horses,

Faculty of Veterinary Medicine, University

of Ljubljana, Ljubljana, Slovenia; ${ }^{7} \mathrm{FEI}$ Quanta,

Eindhoven, The Netherlands; ${ }^{8}$ Laboratory

of Biotechnology, National Institute of

Chemistry, Ljubljana, Slovenia; 'University

of Primorska, Science and Research Centre

of Koper, Koper, Slovenia; ${ }^{10}$ Institute of Cell

Biology, Faculty of Medicine, University of

Ljubljana, Ljubljana, Slovenia; ' 'Department

of Biosciences, Biocity, Åbo Akademi

University, Åbo/Turku, Finland

Correspondence: Veronika Kralj-Iglič Laboratory of Clinical Biophysics, Faculty of

Medicine, University of Ljubljana, Lipičeva 2

SI- 1000 Ljubljana, Slovenia

Tel +386 4l 720766

Fax +386 I43। 5I 27

Email veronika.kralj-iglic@fe.uni-lj.si
Background: Shedding of nanoparticles from the cell membrane is a common process in all cells. These nanoparticles are present in body fluids and can be harvested by isolation. To collect circulating nanoparticles from blood, a standard procedure consisting of repeated centrifugation and washing is applied to the blood samples. Nanoparticles can also be shed from blood cells during the isolation process, so it is unclear whether nanoparticles found in the isolated material are present in blood at sampling or if are they created from the blood cells during the isolation process. We addressed this question by determination of the morphology and identity of nanoparticles harvested from blood.

Methods: The isolates were visualized by scanning electron microscopy, analyzed by flow cytometry, and nanoparticle shapes were determined theoretically.

Results: The average size of nanoparticles was about $300 \mathrm{~nm}$, and numerous residual blood cells were found in the isolates. The shapes of nanoparticles corresponded to the theoretical shapes obtained by minimization of the membrane free energy, indicating that these nanoparticles can be identified as vesicles. The concentration and size of nanoparticles in blood isolates was sensitive to the temperature during isolation. We demonstrated that at lower temperatures, the nanoparticle concentration was higher, while the nanoparticles were on average smaller.

Conclusion: These results indicate that a large pool of nanoparticles is produced after blood sampling. The shapes of deformed blood cells found in the isolates indicate how fragmentation of blood cells may take place. The results show that the contents of isolates reflect the properties of blood cells and their interaction with the surrounding solution (rather than representing only nanoparticles present in blood at sampling) which differ in different diseases and may therefore present a relevant clinical parameter.

Keywords: nanoparticles, nanovesicles, microparticles, microvesicles, cell-cell communication

\section{Introduction}

Early studies reported that "platelet dust" contributing to the coagulation process exists in blood plasma. ${ }^{1}$ It was shown subsequently that nanoparticles are shed from membranes of erythrocytes during storage $\mathrm{e}^{2-4}$ or in vitro in suspensions of cells after the addition of different stimuli. ${ }^{5-7}$ Stimulation of platelets by externally added substances also induces budding and shedding of nanoparticles. ${ }^{8}$ In the literature, nanoparticles created through membrane budding are also called microvesicles, the corresponding process of their formation is called microvesiculation, while platelet-derived nanoparticles are called microparticles. Vesiculation was studied in cancer cells ${ }^{9-13}$ and was shown in vivo to be connected to the impact of cancer-associated coagulopathies. ${ }^{14-17}$ Furthermore, nanoparticles have also been found in isolates from other body fluids, such as synovial fluid of inflamed joints, ${ }^{18}$ pleural fluid, ${ }^{19}$ ascites, ${ }^{19}$ saliva, ${ }^{20,21}$ and urine. ${ }^{22}$ 
Based on accumulated experimental evidence, nanoparticles are now considered membrane-enclosed compartments of the cell interior that are released into the surrounding solution during the final stage of the budding process. They are involved in cell-cell communication ${ }^{23-28}$ and may play a role in the coagulation process inside blood vessels. ${ }^{29}$ Cell-derived nanoparticles should therefore be considered when studying relevant physiological and pathophysiological processes. It has been suggested that determining the concentration and content of nanoparticles in blood may help in the early and low-invasive diagnosis of various common diseases that have extensive medical, ethical, and economic consequences. $^{30-32}$

It would be beneficial to perform diagnosis on samples of peripheral blood instead of on samples obtained by biopsy. It is believed that blood contains circulating nanoparticles ${ }^{33-36}$ carrying information on clinical status and that these nanoparticles can be harvested by isolation. Methods of isolation have been developed consisting mainly of repetitive centrifugation and washing of samples and then assessing the nanoparticles by flow cytometry. ${ }^{35-39}$ The results of numerous clinical studies showed that the concentration of nanoparticles in isolates is increased in patients with a variety of diseases compared with healthy controls..$^{30,40,41}$ However, a method based on the isolation and assessment of nanoparticles from blood that would be of satisfactory repeatability and accuracy and therefore suitable for diagnosis and treatment in clinical practice has not yet been established. Also the mechanisms responsible for budding and vesiculation, including the processes that occur during the isolation procedure, are poorly understood.

Recently, attention has been devoted to the different effects of an isolation procedure on the contents of the isolate (reviewed by Yuana et $\mathrm{al}^{42}$ ). To solve problems arising from preanalytical and analytical issues in the analysis of blood nanoparticles, it is necessary to standardize the isolation protocol. However, we believe that in order to establish assessment of nanoparticles as a clinically relevant method, standardization alone is insufficient, as without an understanding of the underlying mechanisms, parameters that have important effects on the result may be overlooked.

The aim of this study was thus to improve knowledge of the mechanisms and processes taking place during the isolation of nanoparticles and to reveal the morphology and identity of particles in the isolated material. In particular, we tried to answer the question whether nanoparticles found in the isolate are present in blood at sampling or are they created from blood cells during the isolation process. In the latter case, clinical results importantly reflect the vesiculability of cells, mostly platelets. For example, due to interaction of native nanoparticles with platelets, platelets are a potential pool of tumor material and also a source of tumor seeding following removal of a tumor from the body. To diminish the probability of metastases spreading, possible simple therapeutic procedures could be suggested based on the removal of a portion of the platelets after tumor resection and their replacement with platelets from a healthy donor. Further, subsequent attempts to optimize platelet number could complement other therapeutic procedures by slowing down or even stopping tumor progression.

It is therefore likely that information on the source and identity of nanoparticles could shed light on the interpretation of clinical studies involving determination of nanoparticles in blood. Pursuing a study on the origin and identity of nanoparticles in vivo, examining the effects of blood cell fragmentation during isolation should also improve understanding of the basic mechanisms of cell-cell communication and tumor progression.

\section{Materials and methods Subjects}

For the series of experiments on the effect of temperature, blood samples were collected from 56 subjects (students and staff) with no record of disease, after a 12-hour overnight fast. Up to eight samples were processed within a single experiment. The chosen temperature was kept constant during the isolation procedure. The nanoparticles in fresh isolates were counted by flow cytometry immediately after isolation. We discarded 14 samples because the buffer used for washing was found to be contaminated by bacteria. For final analysis we included samples obtained from 42 subjects with no record of disease (29 females and 13 males). In a second set of experiments, blood was collected from seven subjects with no record of disease (four females and three males) after a 12-hour fast. Isolation was first performed at $37^{\circ} \mathrm{C}$ and then a second blood sample was collected from the same subjects in the same consecutive order and the isolation performed at $20^{\circ} \mathrm{C}$. The procedure was then again repeated at $4^{\circ} \mathrm{C}$. The volunteers, consisting of staff and the authors, did not eat or drink over the period of blood sampling and were requested to refrain from physical activity. The isolation procedures were performed at the three different temperatures in a single day so that fresh isolates could be assessed using the same setting of the flow cytometer. Blood sampling was performed by the same nurse in all the experiments that examined the effect of temperature on isolation of nanoparticles. 
Six experiments were performed between 2007 and 2010 to study the correlation between the concentrations of nanoparticles in the isolates and platelets in the blood. A total of 193 subjects with no record of disease were included in these experiments. The volunteers consisted of students of the Faculty of Medicine and Faculty of Veterinary Medicine, University of Ljubljana, staff of collaborating institutions and authors, and 101 participants in the 16th European Veterans Athletic Championships, held in Stadia, Ljubljana, from July 23 to August 3, 2008.

Blood samples for scanning electron microscope imaging of nanoparticles were donated by a patient (female, 60 years) with locally advanced pancreatic cancer, two of the authors with no record of disease (male, 29 years, female, 28 years), and a healthy mare (5 years, $500 \mathrm{~kg}$ ). Mare's blood was used because the large size of the animal allowed collection of a larger volume of blood with minimal discomfort to the animal.

\section{Ethical issues}

All body fluids were acquired with the written consent of the volunteers, patients, and the owner of the mare. The study was approved by the National Ethics Committee. In patients, blood samples for experiments were taken only when blood was needed to be collected for therapeutic reasons, within the same phlebotomy. Likewise, in students, blood samples for experiments were taken when the blood was being collected for obligatory checkups, within the same phlebotomy. For the experiments only, blood was taken from authors, staff, and athlete veterans. For the study of the effect of temperature on the concentration and the size of nanoparticles in isolates, $50 \mathrm{~mL}$ samples of blood were taken from two of the authors and the mare.

\section{Blood sampling}

Blood was collected in $2.7 \mathrm{~mL}$ tubes containing $270 \mu \mathrm{L}$ trisodium citrate at a concentration of $0.109 \mathrm{~mol} / \mathrm{L}$. Evacuated tubes (BD Vacutainers, Becton Dickinson, CA) were used in all the experiments. A 21-gauge needle (length $70 \mathrm{~mm}$, inner radius $0.4 \mathrm{~mm}$, Microlance, Becton Dickinson, $\mathrm{NJ}$ ) was used for blood sampling in all the experiments, with the exception of the experiment in which isolation was performed at three different temperatures in a single day. In that experiment, a 21 gauge needle (Tik d.o.o., Kobarid, Slovenia) was used. During this experiment, the covers of the vacutubes were removed prior to sampling and the blood was allowed to drop freely into the tube. Variation in the acquired volumes did not exceed $15 \%$. In these studies, the tubes were incubated in a rotating centrifuge at the desired temperature and kept in a water bath during handling of the samples. The samples were left in the water bath for several minutes to attain the desired temperature before centrifugation.

\section{Isolation of nanoparticles}

Centrifugation of the samples started within 20 minutes after acquisition of the first sample. In order to separate the cells from plasma, the samples were centrifuged at $1550 \times \mathrm{g}$ for 20 minutes in a Centric 400/R centrifuge (Tehtnica Železniki, Železniki, Slovenia). The upper $250 \mu \mathrm{L}$ of plasma was removed slowly using a tip with a wide opening and placed in a $1.5 \mathrm{~mL}$ Eppendorf tube. The samples were then centrifuged at $17570 \times \mathrm{g}$ for 30 minutes in a Centric $200 / \mathrm{R}$ centrifuge (Tehtnica Železniki). The supernatant $(225 \mu \mathrm{L})$ was discarded and the pellet $(25 \mu \mathrm{L})$ resuspended in $225 \mu \mathrm{L}$ citrated phosphate-buffered saline. The samples were centrifuged again at $17570 \times \mathrm{g}$ for 30 minutes, the supernatant $(225 \mu \mathrm{L})$ discarded and the pellet $(25 \mu \mathrm{L})$ resuspended in $75 \mu \mathrm{L}$ of citrated phosphate-buffered saline.

\section{Flow cytometric analysis}

Flow cytometric analysis was performed using an Altra Flow Cytometer (Beckman Coulter Inc, Fullerton, CA) with a $488 \mathrm{~nm}$ water-cooled laser. The Coulter EXPO32 software was used for data acquisition and analysis of the results. The presence of particles (residual cells and nanoparticles) was determined by forward and side scatter parameters. The flow count of fluorospheres (10 $\mu \mathrm{m}$, Beckmann Coulter) at a known concentration $\left(1 \times 10^{6} / \mathrm{mL}\right)$ was used to determine the concentration of nanoparticles. At least 10,000 events were recorded in each sample analysis. The size of the particles in the sample was estimated by the mean intensity of the forward scattered light.

\section{Scanning electron microscopy}

The nanoparticles were suspension-fixed in $1 \%$ glutaraldehyde dissolved in phosphate-buffered saline/citrate buffer for 60 minutes at $22^{\circ} \mathrm{C}$, post-fixed for 60 minutes at $22^{\circ} \mathrm{C}$ in $1 \%$ $\mathrm{OsO}_{4}$ dissolved in $0.9 \% \mathrm{NaCl}$, dehydrated in a graded series of acetone/water (50\%-100\%, v/v) and critical point dried. At the Åbo Akademi University, the samples were gold-sputtered, and examined using a LEO Gemini 1530 (LEO, Oberkochen, Germany) scanning electron microscope, while at FEI Quanta, the samples were iridium-coated, and examined using a Quanta TM 250 FEG (FEI, Hillsboro, OR) scanning electron microscope. Drying may have caused shrinking of objects up to $20 \%$, the effect being more pronounced for larger objects (cells). 


\section{Determination of theoretical shapes}

The shapes of the nanoparticles at equilibrium were calculated by minimization of the membrane free energy, ${ }^{43}$ taking into account possible orientational ordering of membrane constituents in highly anisotropically curved regions, and also assuming rotational symmetry of nanoparticles with respect to the symmetry axis. ${ }^{44,45}$ Membrane area, enclosed volume, and average mean curvature were kept constant during minimization. The system of differential equations was solved numerically, as described by Iglič et al. ${ }^{45}$

\section{Determination of average size of nanoparticles}

The size of the nanoparticles was estimated from micrographs by measuring the linear dimension of the nanoparticle cross-sections, inclined at an angle of $45^{\circ}$ with respect to the coordinate system determined by the principal axes. All clearly visible shapes within a frame were considered. The measurements were carried out using Corel Draw software (Corel Corporation, Ottawa, Canada).

\section{Results}

Figure 1 shows a typical scanning electron micrograph of an isolate from the blood of a healthy human donor, exhibiting a mass of nanoparticles and many residual erythrocytes. Figure 2 shows scanning electron micrographs of chosen regions of an isolate from the blood of a healthy human donor. Heparin (A-D) and trisodium citrate (E, F) were used

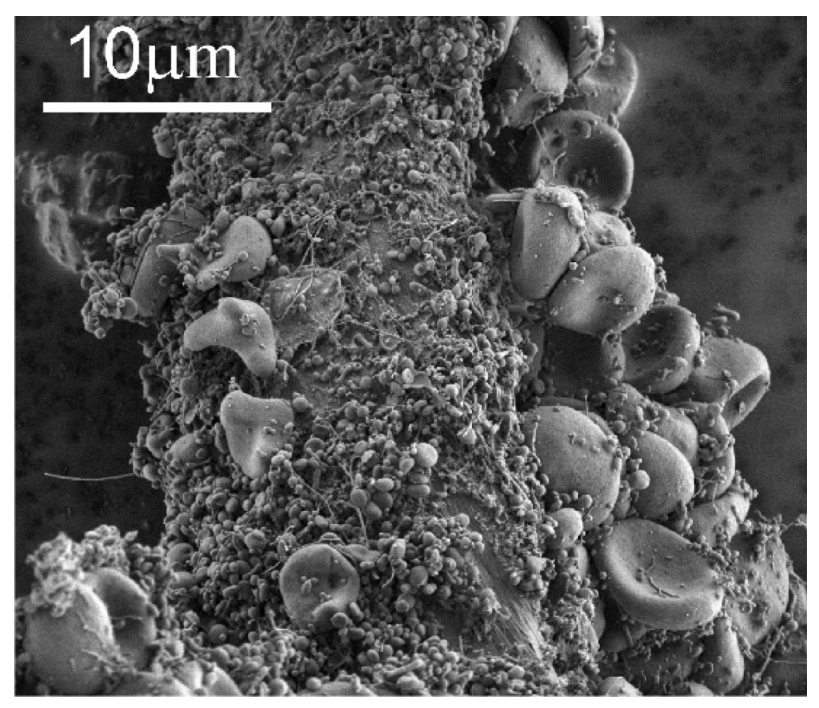

Figure I Scanning electron micrograph of an isolate from peripheral blood of a healthy human donor (male, 28 years). A mass of microparticles and numerous residual erythrocytes can be seen. The image was taken using a Quanta TM 250 FEG (FEl, Hillsboro, OR) scanning electron microscope at FEl Quanta, Eindhoven, The Netherlands, by applying $1.5 \mathrm{kV}$.
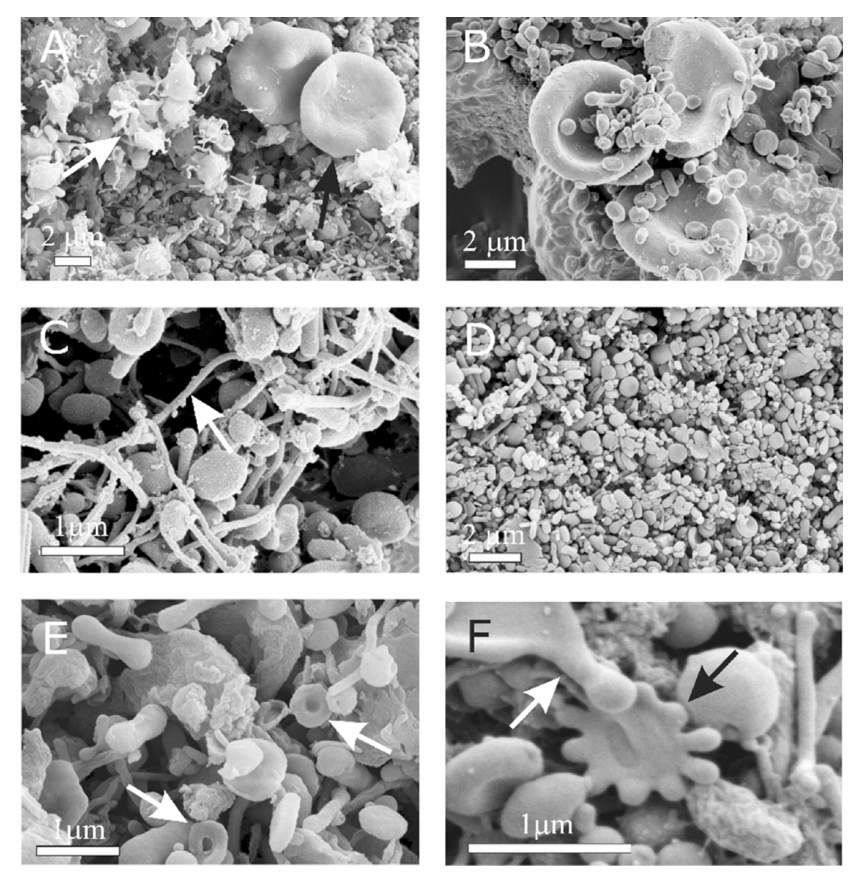

Figure 2 Scanning electron micrograph of chosen regions of an isolate from peripheral blood of a healthy human donor (male, 28 years). In addition to numerous nanoparticles which are present in all the pictures, erythrocytes (A-black arrow, B), activated platelets ( $\mathbf{A}$ - white arrow), tubules $(\mathbf{C})$, tori (E - white arrows), starfish ( $\mathbf{F}$ - black arrow) and a deformed erythrocyte exhibiting protrusion with a bulbous end (F - white arrow) were observed. (A - D) images taken using a LEO Gemini I530 (LEO, Oberkochen, Germany) scanning electron microscope by applying $8 \mathrm{kV}$ (A, C, and D) and $2.7 \mathrm{kV}$ (B), at Åbo Akademi University, Åbo/Turku. Images E and $\mathbf{F}$ taken by Quanta TM $250 \mathrm{FEG}$ (FEl, Hillsboro, Oregon, OR) scanning electron microscope at FEI Quanta, Eindhoven, The Netherlands, by applying I.5 kV.

as the anticoagulants. Numerous nanoparticles can be seen, while residual cells such as erythrocytes (A, black arrow) and activated platelets (A, white arrow) were also present in the isolate. The nanoparticles had diverse shapes and sizes. Tubular structures of different lengths were found (B-F), which were more abundant with heparin than with trisodium citrate as the anticoagulant. Peculiar structures such as a torus (E, white arrows) and starfish (F, black arrow) were found. The white arrow in Figure 1F identifies a cell which formed protrusions with bulbous ends.

Figure 3 shows some characteristic nanoparticle shapes found in an isolate from the blood of a patient with pancreatic cancer (female, 60 years). Some rather large fragments with a low volume:area ratio (A, B), nanosized discocytes (C), and dumb-bell shapes (D) can be seen. Comparison with shapes obtained by minimization of the membrane free energy (E, F) showed good agreement. This indicates these particles are membrane-enclosed entities without an internal structure and can therefore be described as vesicles.

To increase the accuracy of the size determinations, samples of nanoparticles isolated from the same blood were divided into two parts, with one imaged at Åbo Akademi University, 

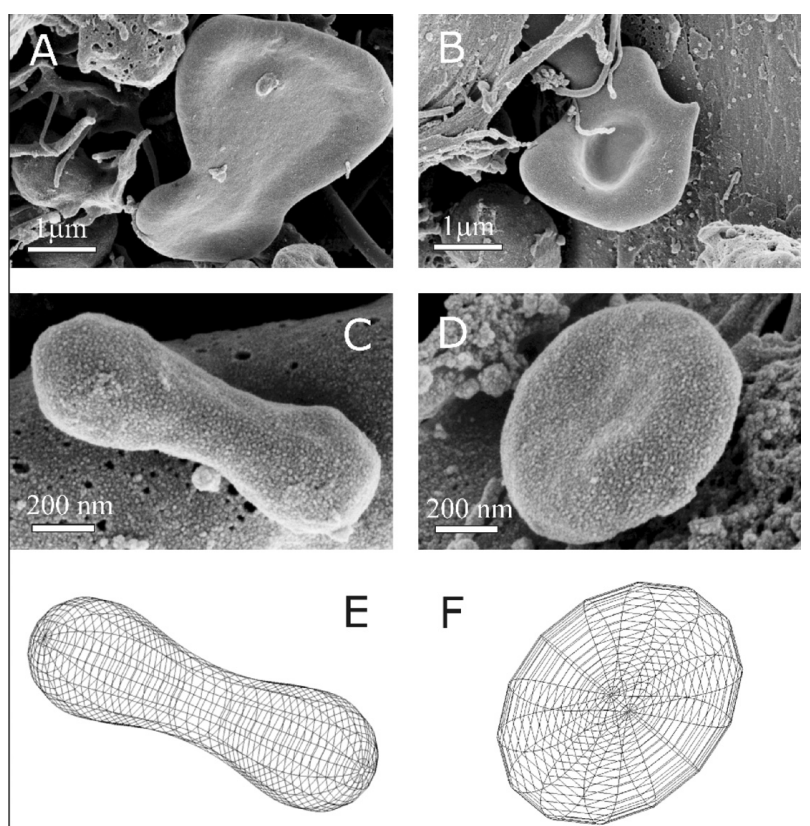

Figure 3 Representative characteristic shapes of nanoparticles found in an isolate from the blood of a patient with pancreatic cancer (female, 60 years). Shapes include shizocytes (A and $\mathbf{B})$, dumbbell $(\mathbf{C})$, submicron discocyte (D), and the corresponding shapes calculated by minimization of the membrane free energy (E and $\mathbf{F})$. The shape in panel $\mathbf{E}$ was obtained for a relative volume $v=36 \pi V^{2 / 3} / A^{3 / 2}=0.65$, where $V$ is the volume of the vesicle, $A$ is the surface area of the vesicle and for the relative average mean curvature $\langle h\rangle=1 / 2 \mathrm{~A} \int\left(C_{1}+C_{2}\right) \mathrm{d} A=1.32$, where $C_{1}$ and $C_{2}$ are the two principal curvatures at a chosen point of the membrane surface and integration is performed over the entire surface of the vesicle $A$. For the shape in panel $F, v=0.55$ and $\langle h\rangle=1.055$. Intrinsic principal curvatures were equal to 0 for both shapes. The images were taken using a LEO Gemini 1530 (LEO, Oberkochen, Germany) scanning electron microscope by applying $8 \mathrm{kV}$ at Åbo Akademi University, Åbo/ Turku, Finland.

Åbo/Turku, Finland and the other at FEI Quanta, Eindhoven, Netherlands. The isolations were performed at room temperature $\left(25^{\circ} \mathrm{C}\right)$. Drying and gold-sputtering/iridium-coating was performed at the two laboratories. The effective diameter of the nanoparticles measured in blood isolates by both laboratories was about $300 \mathrm{~nm}$ (Table 1). The difference between the results of the two laboratories was not statistically significant.

Regarding the origin and mechanisms of nanoparticle generation in the isolates, the structures observed in Figure 4A

Table I Size (diameter) of nanoparticles isolated from the same human blood sample as observed by two different scanning electron microscopes

\begin{tabular}{llll}
\hline Sample & NP $(\mathbf{n})$ & Diameter \pm SD $(\mathbf{n m})$ & $\mathbf{P}$ \\
\hline $\begin{array}{l}\text { Human blood } \\
\text { (FEl Quanta) }\end{array}$ & 85 & $321 \pm 130$ & 0.28 \\
Human blood $(\AA A))$ & 101 & $302 \pm 131$ & \\
\hline
\end{tabular}

Notes: Average size of nanoparticles determined by measuring the linear dimensions of nanoparticles imaged by the scanning electron microscopes at FEI Quanta, Eindhoven, Netherlands, and at Åbo Akademi University, Abo/Turku, Finland (ÅA). The nanoparticles were isolated at room temperature. The $P$ value of the difference between the mean sizes obtained by the two microscopes was calculated. Abbreviations: NP, nanoparticles; SD, standard deviation.
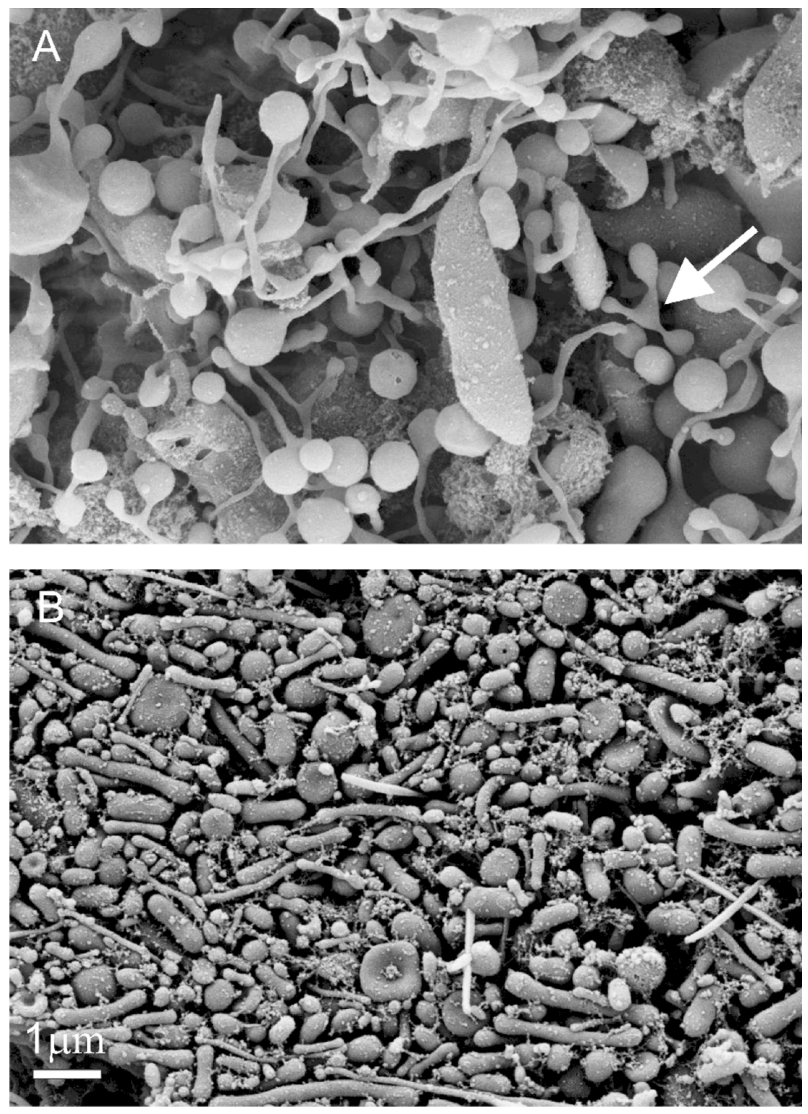

Figure 4 Deformation of cell-derived material obtained by isolation procedure Deformed cells from the blood of a healthy mare (aged five years) exhibit protrusions connected by thin necks, which were torn eventually to yield membrane-enclosed cell fragments $(\mathbf{A})$. Close to the tube wall, the shear forces in the centrifuge are high and therefore the cell fragments in the isolate from the blood of a healthy human donor (male, 28 years) are elongated and exhibit preferential orientation (B). The images were taken using a LEO Gemini 1530 (LEO, Oberkochen, Germany) scanning electron microscope by applying $8 \mathrm{kV}$ at Åbo Akademi University, Åbo/ Turku, Finland.

indicate that fragmentation of blood cells occurs during the process. Panel A shows the presence of tubular structures connected to distal bulbous parts (see also Figure 3B). Thin necks were formed (white arrow), which could have be torn by centrifugal shear stress to produce the rather large cell fragments found in the isolates. It is evident that the particles which we considered to be nanoparticles (Figures 1 and 2) attained the sizes and shapes indicated by those of the precursors seen in deformed cells (Figure 4A).

The micrograph shown in Figure 4B was taken close to the interface between the isolate and the epruvette wall, where the shear force was expected to be greatest. Numerous elongated shapes, preferentially oriented in a particular direction can be seen, indicating that shear stress in the centrifuge affects nanoparticle shape.

The process of nanoparticle isolation was studied at different temperatures that were kept constant throughout 
the isolation process using a water-bath and a temperatureregulated centrifuge. The first population study including 42 healthy volunteers showed that the concentration of nanoparticles in the isolates determined by flow cytometry was lower at higher temperatures (Table 2). Because the accuracy of flow cytometry in determining the nanoparticle concentration is rather poor, a further study was designed to obtain a more decisive result. In the second study, all the samples were measured using the same setting of the flow cytometer. However, this limited the second study to a small number of subjects. In the second study, blood was taken from the same subjects for all three temperatures investigated. Blood was acquired by free flow to minimize activation of platelets in the needle that could have been an additional source of difference between the samples. The results of the second study confirmed the results of the first study that the concentration of nanoparticles in the isolates was higher at lower isolation temperatures (Table 3). However, we observed that the time needed to acquire the required volume of blood by free flow differed markedly between subjects and also in repetitive acquisitions from the same subject, indicating corresponding differences in shear stress. Because this could be a source of considerable difference in the nanoparticle concentrations in isolates, we performed another analysis of the results using only data on blood samples from each subject that differed in collection time by less than 15 seconds. The data which were omitted are marked with asterisks in Table 3. In subject 4, all three collection times differed by more than 15 seconds, so we retained only one result. The results retained show that in all subjects the concentration of nanoparticles in the isolates decreased gradually with increasing temperature during isolation (Table 3).

We also visualized the platelets in platelet-rich plasma at different temperatures in a healthy human (Figure 5A-C) and a healthy mare (Figure 5D-F). The respective nanoparticles isolated from these samples are shown in Figure 5G-I. At temperatures below room temperature, platelet shapes

Table 2 Concentration of nanoparticles obtained by isolating nanoparticles from blood of 42 donors with no record of disease, at different temperatures

\begin{tabular}{|c|c|c|c|c|}
\hline $\mathbf{T}\left({ }^{\circ} \mathrm{C}\right)$ & Subjects (n) & NP/spheres \pm SD & $\begin{array}{l}P \text { value vs } \\
37^{\circ} \mathrm{C}(\mathrm{P})\end{array}$ & $\begin{array}{l}P \text { value vs } \\
40^{\circ} \mathrm{C}(\mathrm{P})\end{array}$ \\
\hline 30 & 12 & $2.90 \pm 1.85$ & $0.01(0.64)$ & $0.001(0.82)$ \\
\hline 37 & 17 & $1.31 \pm 1.45$ & I & $0.31(0.16)$ \\
\hline 40 & 13 & $0.87 \pm 0.45$ & - & 1 \\
\hline
\end{tabular}

Notes: Statistical significance of differences $(P)$ and corresponding statistical power $(P)$ are shown. NP indicates nanoparticles and T indicates temperature.

Abbreviations: NP, nanoparticles; SD, standard deviation; vs, versus exhibited filopodia and distortion compared with the resting discocyte shape in the human and the mare (Figure 5B and $\mathrm{E})$. The shapes of the nanoparticles were the same at all temperatures (Figure 5G-I). However, their mean size measured in human by flow cytometry (Table 4) and in the mare by scanning electron microscopy (Table 5) was dependent on the isolation temperature. As shown in Table 4, the increase in size with increasing temperature was gradual in all subjects (all data included). The differences between the size of nanoparticles isolated from mare's blood at $20^{\circ} \mathrm{C}$ and $37^{\circ} \mathrm{C}$ and at $30^{\circ} \mathrm{C}$ and $37^{\circ} \mathrm{C}$ were considerable $(29 \%$ and $22 \%$, respectively). These differences were statistically significant $(P<0.0001)$ and of sufficient power $(P$ values at $\alpha=0.05$ were 0.99 and 0.96 , respectively) (Table 5). All the differences between the mean size of nanoparticles isolated from human blood at 4,20 , and $37^{\circ} \mathrm{C}$ and measured by flow cytometry were shown to be statistically significant (Table 4). The large size of nanoparticles in the isolates, the shapes of the intermediate structures leading to isolated material, and the sensitivity of the concentration and mean size of nanoparticles to external parameters such as temperature indicate that a large pool of nanoparticles were created after blood sampling.

\section{Discussion}

Our previous experience with microvesiculation was based on the process of cell budding observed in vitro. ${ }^{7,8}$ In experiments with artificially induced vesiculation of erythrocytes, the buds observed on the top of the spicules corresponded in shape and size to the nanoparticles found in isolates (Figure 6A and C). Because budding and vesiculation are common processes in all cells, we expected that nanoparticles created in vivo would also be present in blood and could be detected by isolation. However, we experienced poor accuracy and repeatability in the isolation procedure (ie, nanoparticle concentration) during our attempts to perform clinical and population studies (not shown), leading us to pose the following questions: Are the nanoparticles found in isolates present in blood in vivo? What are the processes leading to the formation of nanoparticles in isolates? What is the content of the isolates? What is the identity of nanoparticles in isolates with respect to the mother cell(s)?

To answer these questions we used a combination of methods to visualize the isolates and cell sediments and performed theoretical analyses of shapes, flow cytometry, and population studies. Our results indicate that isolates from blood contain a mass of submicron-sized particles that have the characteristic shapes of membrane-enclosed fragments with no internal 
Table 3 Concentration of nanoparticles isolated from human blood at different temperatures and time of blood sampling for seven subjects

\begin{tabular}{|c|c|c|c|c|c|c|}
\hline Subject & $\begin{array}{l}\text { NP/spheres } \\
4^{\circ} \mathrm{C}\end{array}$ & $\begin{array}{l}\text { Time (seconds) } \\
4^{\circ} \mathrm{C}\end{array}$ & $\begin{array}{l}\text { NP/spheres } \\
\mathrm{T}=20^{\circ} \mathrm{C}\end{array}$ & $\begin{array}{l}\text { Time (seconds) } \\
20^{\circ} \mathrm{C}\end{array}$ & $\begin{array}{l}\text { NP/spheres } \\
\mathrm{T}=37^{\circ} \mathrm{C}\end{array}$ & $\begin{array}{l}\text { Time (seconds) } \\
37^{\circ} \mathrm{C} \\
\end{array}$ \\
\hline I & 0.54 & 64 & $0.58 *$ & $110 *$ & 0.35 & 68 \\
\hline 2 & 0.29 & 55 & 0.22 & 57 & $0.29 *$ & $40^{*}$ \\
\hline 3 & 1.21 & 104 & $0.78 *$ & $114 *$ & 0.21 & 90 \\
\hline 4 & $9.34 *$ & $72^{*}$ & $0.23 *$ & $163^{*}$ & 0.42 & 55 \\
\hline 5 & 0.58 & 76 & 0.32 & 81 & 0.17 & 86 \\
\hline 6 & $0.26 *$ & $108^{*}$ & 0.55 & 82 & 0.28 & 84 \\
\hline 7 & $0.90 *$ & $64^{*}$ & 0.38 & 81 & 0.23 & 80 \\
\hline Average & 1.88 & 78 & 0.43 & 98 & 0.29 & 72 \\
\hline Average $^{\dagger}$ & 0.65 & 75 & 0.38 & 75 & 0.28 & 77 \\
\hline
\end{tabular}

Notes: Data differing in blood sampling time longer than 15 seconds are marked by asterisks. The average marked by a cross was obtained by omitting the data marked by asterisks.

structure. However, these particles are rather large (on average $>300 \mathrm{~nm}$ ) compared with the nanovesicles obtained from erythrocyte budding in vitro (on average $<180 \mathrm{~nm}$ ) as shown in Figure 6. To provide more independent reliable evidence on the size and morphology of nanoparticles, blood samples were divided into two parts which were imaged using two different scanning electron microscopes. The results were in qualitative and quantitative agreement (Table 1). We found that isolates may also contain many residual blood cells, such as erythrocytes (Figures 1,2A and B), leukocytes,
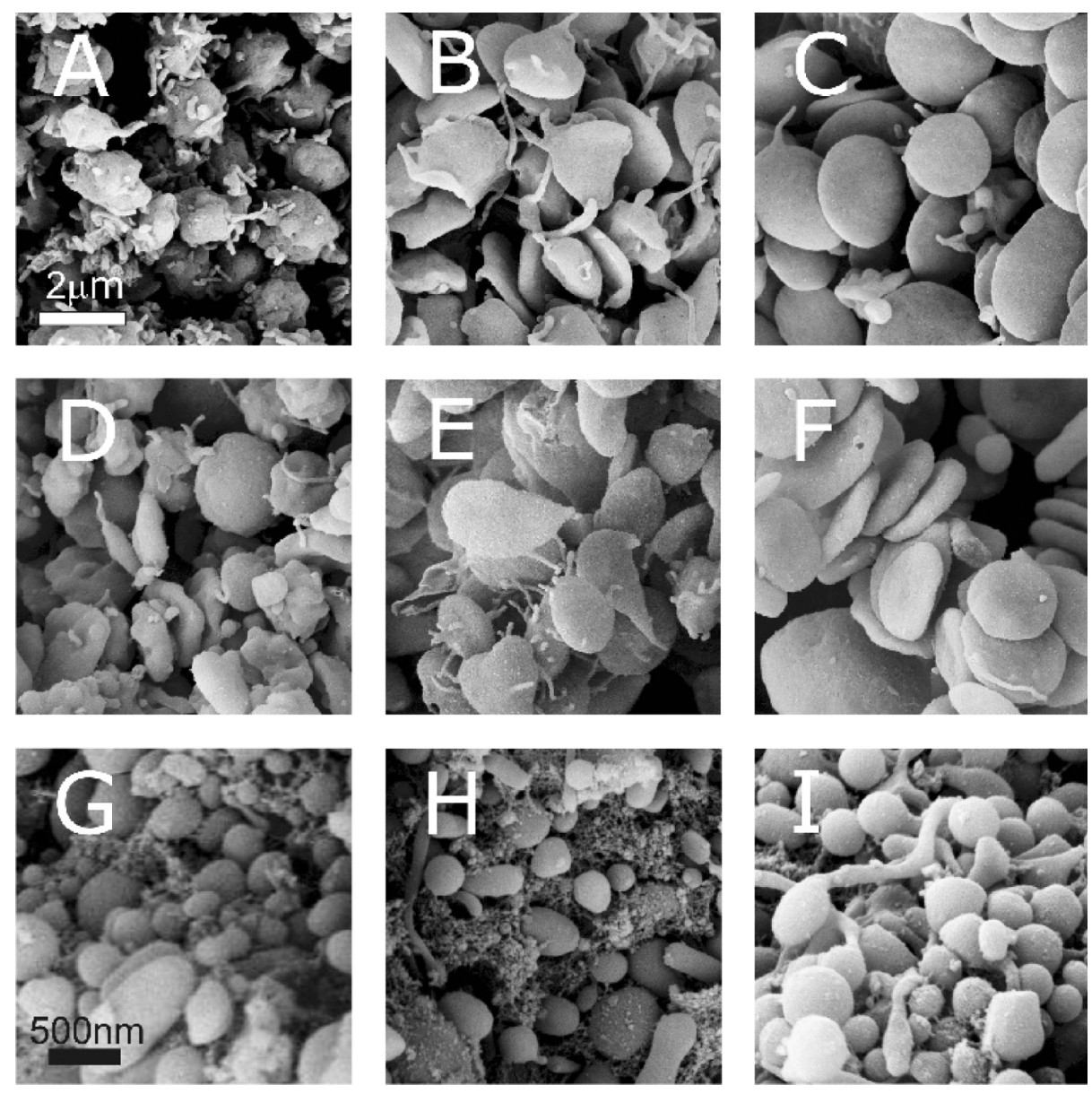

Figure 5 Platelets and nanoparticles at different temperatures. Nanoparticles were isolated from platelet-rich plasma of a healthy human donor (female, 28 years) at different temperatures $\left(\mathbf{A}: 4^{\circ} \mathrm{C}, \mathbf{B}: 20^{\circ} \mathrm{C}, \mathbf{C}: 37^{\circ} \mathrm{C}\right)$, platelets from platelet-rich plasma of a mare $\left(\mathbf{D}: 4^{\circ} \mathrm{C}\right.$, E: $\left.20^{\circ} \mathrm{C}, \mathbf{F}: 37^{\circ} \mathrm{C}\right)$, nanoparticles isolated from blood of the mare $\left(\mathbf{G}: 4^{\circ} \mathrm{C}\right.$, H: $20^{\circ} \mathrm{C}$, I: $37^{\circ} \mathrm{C}$ ). The images were taken using a LEO Gemini I 530 (LEO, Oberkochen, Germany) scanning electron microscope by applying 8 kV at Åbo Akademi University, Åbo/Turku, Finland. 
Table 4 Mean of the parameter representing flow cytometric measurement of light scattering in the forward direction (relative units) at different temperatures

\begin{tabular}{llll}
\hline Subject & $\begin{array}{l}\text { Mean FS } \\
\mathbf{T}=\mathbf{4}^{\circ} \mathbf{C}\end{array}$ & $\begin{array}{l}\text { Mean FS } \\
\mathbf{T}=\mathbf{2 0} \mathbf{0}^{\circ} \mathbf{C}\end{array}$ & $\begin{array}{l}\text { Mean FS } \\
\mathbf{T}=\mathbf{3 7} \mathbf{0}^{\circ} \mathbf{C}\end{array}$ \\
\hline 1 & 1.2 & 2.1 & 2.1 \\
2 & 1.4 & 1.7 & 2.7 \\
3 & 0.9 & 1.3 & 2.3 \\
4 & 0.9 & 1.8 & 2.0 \\
5 & 1.5 & 2.1 & 2.4 \\
6 & 1.7 & 1.9 & 2.4 \\
7 & 1.3 & 1.8 & 1.9 \\
Average & 1.3 & 1.8 & 2.3 \\
\hline
\end{tabular}

Note: This parameter represents the mean size of nanoparticles. Abbreviation: FS, forward scatter.

activated platelets (Figure 2A), and nanotubular structures with bulbous ends which appear as hollow tubes attached to the fibrin network (Figure 2C). This information is useful for gating of the population events detected by flow cytometry.

The concentration of nanoparticles in the isolates was lower and their size larger at higher isolation temperatures (Tables 2-5). In addition to affecting chemical reactions, temperature also affects nonspecific biophysical processes. The following equation taking into account centrifugal force, buoyancy, and Stokes law governs the sedimentation velocity of a spherical particle:

$$
v=\Delta \rho d^{2} a / 18 \eta
$$

where $\Delta \rho$ is the difference in densities of the nanoparticles and the medium (plasma), $d$ is the effective diameter of the NP, $a$ is the acceleration of the centrifugal force created in the centrifuge rotor, and $\eta$ is the viscosity of the medium.

It follows from equation (1) that both native nanoparticles possibly present in blood, and nanoparticles which were possibly created after sampling, would sediment more slowly at lower temperatures due to the higher viscosity of plasma

Table 5 Size of nanoparticles isolated from mare's blood at different temperatures (diameter measured from scanning electron microscope images taken at Åbo Akademi University, Åbo/Turku, Finland)

\begin{tabular}{|c|c|c|c|c|}
\hline $\mathrm{T}\left({ }^{\circ} \mathrm{C}\right)$ & $\begin{array}{l}\text { NP } \\
\text { measured (n) }\end{array}$ & $\begin{array}{l}\text { Diameter } \pm \\
\text { SD }(\mathbf{n m})\end{array}$ & $\begin{array}{l}P \text { value vs } \\
30^{\circ} \mathrm{C}(\mathrm{P})\end{array}$ & $\begin{array}{l}P \text { value vs } \\
37^{\circ} \mathrm{C}(\mathrm{P})\end{array}$ \\
\hline 20 & 44 & $273(82)$ & $0.331(0.166)$ & $\begin{array}{l}<0.000 \text { I } \\
(0.99)\end{array}$ \\
\hline 30 & 51 & $291(101)$ & I & $\begin{array}{l}<0.000 \text { I } \\
(0.95)\end{array}$ \\
\hline 37 & 67 & $362(100)$ & & I \\
\hline
\end{tabular}

Abbreviations: NP, nanoparticles; SD, standard deviation.

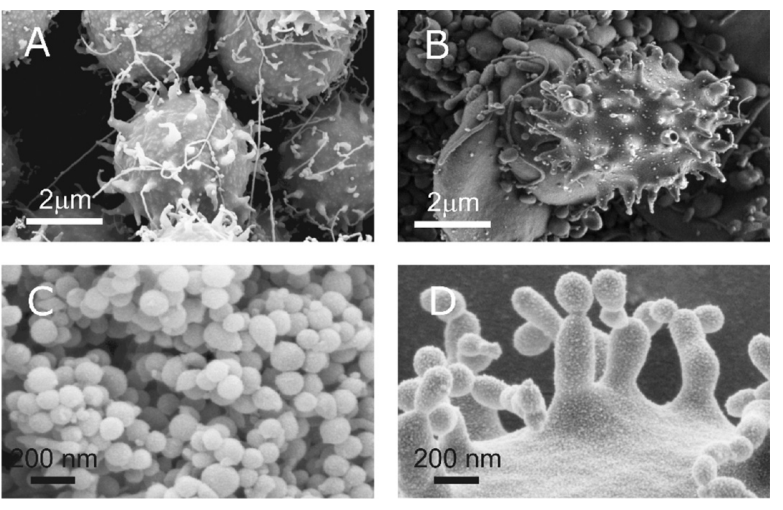

Figure 6 Budding of membranes. (A) Budding of erythrocytes induced by adding detergent to the suspension of erythrocytes (scanning electron microscopy performed at Åbo Akademi University, Åbo/Turku, Finland). (B) A budding erythrocyte found in an isolate from human blood with numerous nanoparticles (scanning electron microscopy performed at FEl Quanta, Eindhoven, The Netherlands). (C) Nanoparticles isolated from suspension of erythrocytes with added detergent. (D) Precursors of nanoparticles appearing at the top of the echinocyte spicules. (A, C, and $\mathbf{D}$ images taken using a LEO Gemini 1530 (LEO, Oberkochen, Germany) scanning electron microscope by applying $8 \mathrm{kV}$, at Åbo Akademi University, Åbo/Turku, Finland. Image (C) taken by Quanta TM 250 FEG (FEl, Hillsboro, OR) scanning electron microscope at FEl Quanta, Eindhoven, The Netherlands, by applying I.5 kV). Image in Panel B was taken from Šuštar et al..$^{46}$

at these temperatures. Therefore, clearance of cells from the upper part of the tube used to isolate nanoparticles during the first centrifugation is less effective. Cells remaining in this compartment may shed nanoparticles into the sample during centrifugation. We also observed that platelets become activated below room temperature and increasingly deform with decreasing temperature (Figure 5). Activation of platelets causes changes in the integrity of their cytoskeleton and renders the cells prone to fragmentation, thereby contributing to an increased number of nanoparticles in the isolates at lower temperatures. All the above facts explain the lower concentration of nanoparticles we observed in isolates prepared at higher temperature (Tables 2 and 3). Because the effects on nanoparticle concentration of factors that determine the sedimentation speed of existing nanoparticles and the creation of new nanoparticles during the first step of isolation are synergistic, we could not distinguish between their respective contributions to the overall effect. However, the mean size of the nanoparticles was also different at different isolation temperatures. If isolation primarily yields the nanoparticles that were present in blood in vivo, the average size of nanoparticles present in the upper compartment of plasma after the first centrifugation should be smaller at higher temperatures according to equation (1), and the clearance of larger nanoparticles would be more effective. Assuming that the second and the third centrifugation collects nanoparticles in the pellet, the average size of nanoparticles in isolates would be expected to be smaller at higher temperatures. In contrast, 
we observed that nanoparticles are larger when isolated at higher temperatures (Tables 4 and 5).

Staining with antibodies for platelet origin (anti-CD42bPE and anti-CD31FITC positive events) showed that the majority $(70 \%)$ of nanoparticles contain receptors derived from platelets. ${ }^{46}$ This finding agrees with previous reports. ${ }^{40}$ However, it was unclear how particles sized $300 \mathrm{~nm}$ or greater that were mainly quasiglobular-shaped could be obtained by budding of activated platelets. For example, the filopodia of activated platelets are thinner (Figure 5A-F), so if these were pinched off from the mother cell, the nanoparticles produced would be evidently smaller and/or tubular. Despite the considerable evidence that platelets are the main origin of nanoparticles in blood isolates, ${ }^{40}$ by using the described protocol for isolation we found no correlation between the number of platelets in blood and the number of nanoparticles found in blood isolates (Table 6). This relationship should be expected if the nanoparticles found in isolates were predominantly present in blood in vivo.

Platelets are affected by shear stress in the needle during blood sampling. Assuming laminar stationary flow of a Newtonian viscous fluid and the validity of the PoiseuilleHagen law, the velocity in the direction of flow is subject to a parabolic profile that is dependent on the distance from the center of the needle. During free flow, the flow rate is determined by the difference between the pressure in the vein and atmospheric pressure which results in the rather slow dripping of blood. The gradient of velocity determines the shear force that is greatest near the inner wall of the needle:

$$
\mathrm{F}=8 \eta V l / t R^{2}
$$

where $V$ is the volume of blood, $l$ is the length of the needle, $t$ is the time required to acquire the blood, and $R$ is the inner radius of the needle.

The variation in time that reflects the speed of blood in the needle may be the cause of the poor accuracy when determining the concentration of nanoparticles. In the second experiment that assessed the temperature dependence of the nanoparticle concentration, we measured the time required to acquire $2.9 \mathrm{~mL}$ of blood. Because it is still unclear how shear stress affects the concentration of nanoparticles in isolates, we omitted those measurements from the final analysis that differed in collection time by more than 15 seconds. The remaining data showed monotonous behavior in the relationship of nanoparticle concentration on temperature in all seven subjects. The lower pressure in evacuated tubes used for blood sampling results in greater flow and therefore shorter times to acquire the same volume of blood, with a correspondingly larger velocity of blood and shear stresses in the needle. A thorough theoretical and experimental analysis of blood flow in the needle is required to optimize blood sampling.

The isolation procedure depends on external parameters that cannot be kept constant with the existing equipment and protocol. This explains the poor repeatability and accuracy of nanoparticle isolation in different experiments and certainly adds to the lack of correlation we observed between the concentration of nanoparticles in isolates and the concentration of platelets in blood. However, another explanation consistent with the above findings is that the majority of nanoparticles are created after blood sampling, and accordingly the results reflect the properties and composition of blood cells and plasma. For example, membrane and plasma constituents may affect membrane curvature, ${ }^{47-49}$ which is the basic mechanism underlying the budding and vesiculation of membranes. Controlled manipulation of the size of the cell fragments (nanoparticles) could be advantageous, as avoiding production of fragments of the size of immune complexes (mean diameter approximately $150 \mathrm{~nm}$ ) may avoid problems with artefacts when measuring the nanoparticle concentration by flow cytometry. ${ }^{50}$

The properties of blood cells and plasma may have an important influence on the state of the isolate. One can interpret the alteration of blood cells in cancer patients by

Table 6 Correlation between nanoparticle concentration in isolate and platelet concentration in blood in six experiments involving healthy human donors

\begin{tabular}{llllll}
\hline Experiment & Subjects $(\mathbf{n})$ & Platelets \pm SD [1 $\left.\mathbf{0}^{9} / \mathbf{L}\right]$ & NP/spheres \pm SD & Pearson coefficient & Statistical significance $(\boldsymbol{P})$ \\
\hline $\mathrm{I}$ & 64 & $250 \pm 46$ & $0.67 \pm 0.33$ & 0.15 & 0.38 \\
2 & 25 & $250 \pm 45$ & $0.66 \pm 0.33$ & 0.11 & 0.63 \\
3 & 24 & $244 \pm 51$ & $2.52 \pm 1.86$ & 0.23 & 0.35 \\
4 & 23 & $1.82 \pm 1.03$ & -0.03 & 0.88 \\
5 & 25 & $235 \pm 69$ & $1.03 \pm 0.55$ & -0.06 & 0.78 \\
6 & 28 & $238 \pm 53$ & $1.11 \pm 0.72$ & 0.02 & 0.90 \\
\hline
\end{tabular}

Abbreviations: NP, nanoparticles; SD, standard deviation. 
the presumable integration of native tumor cell-derived nanoparticles into their membranes. Therefore, blood cells, especially platelets, may convey tumor cell material to distal cells and render it functional by inducing processes in these cells. ${ }^{51-53}$ It has been suggested that metastases are seeded by tumor cells and that the probability of this happening is greater when microemboli composed of tumor cells and platelets travel slowly in capillaries, thereby enhancing the probability of tumor cells entering the tissues through the endothelium. ${ }^{54}$ Platelets are known to adhere to tumor cells and shield them from attack by leukocytes. ${ }^{54}$ Indeed, there is evidence that platelets support tumor metastasis ${ }^{54}$ and platelet counts are related to prognosis in cancer patients. ${ }^{55,56}$ Because tumor cells are unlikely to be found in vivo in patients, the above hypothesis is based on cancer induction by injection of tumor cells directly into the blood of animals. On the other hand, it appears likely that native nanoparticles shed by cancer cells are the origin of metastases, either as vehicles or as mediators of transport by mobile cells, most probably platelets. It has been reported that nanoparticles shed from cells interact with other cells, ${ }^{57-60}$ so it is possible that material shed from blood cells is exchanged constantly between cells via native nanoparticles. Because platelets are prone to vesiculate, the material shed during this process is likely to be found in other cells, such as erythrocytes, leukocytes, and endothelial cells. The nanoparticle-mediated exchange of material between cells may explain the intriguing data of Boilard et al, who found platelet-derived material, but no platelets, in the synovial fluid of patients with rheumatoid arthritis. ${ }^{58}$

Figure 6 shows budding of erythrocytes and the respective isolated microvesicles. In the sample shown in Panel A, budding was induced by adding echinocytogenic molecules to the suspension, while in the sample shown in Panel B, echinocytosis took place during isolation due to mechanical and thermal stress. Regardless of the mechanism, the shapes of the echinocytes and the buds are similar. It would therefore be expected that erythrocyte-derived microvesicles (Panel C) are considerably smaller than the nanoparticles observed in Figures 1-5 and would not have been detected by flow cytometry.

The shapes shown in Figure $3 \mathrm{E}$ and $\mathrm{F}$ were calculated theoretically by minimization of the membrane free energy as described elsewhere. ${ }^{45}$ It was assumed that the vesicle has no internal structure and that its shape was determined by the properties of the membrane. The membrane free energy was derived using the energies of individual membrane constituents and for simplicity, it was assumed that all constituents were equal. Entropic effects were also taken into account. The free energy obtained was determined by a thermodynamic expression for the elastic energy using statistical physics methods. ${ }^{44,45}$ The calculated shapes of minimal elastic energy were characterized by a high degree of symmetry and smooth contours that avoided strong bending, which is energetically unfavorable. Vesicles undergo fluctuations in shape due to thermal effects, so their instantaneous shapes deviate somewhat from the ideal morphology predicted by theory and calculations. Nevertheless, matching of the appearance of the observed and the calculated shapes of blood-derived nanoparticles was excellent in our study (Figure 3C-F). It can also be seen in Figures 1 and 2 that the contours of the nanoparticles are highly symmetrical and smooth, indicating that these shapes correspond to microvesicles with no internal structure. Calculation of shape changes in the sedimentation field may be of considerable help in revealing the mechanisms of cell budding and vesiculation during centrifugation and remains a task for future research.

\section{Conclusion}

By visualizing isolates and comparing theoretically obtained shapes with observed shapes, we showed that submicron-sized particles were present in the material obtained from blood by centrifugation and washing. However, analysis of the nanoparticle shapes and sizes obtained indicates that a large pool of these nanoparticles is composed of cell fragments, which were most likely formed as a result of mechanical stress during centrifugation and also thermal stress during isolation. The concentration, size, and identity of nanoparticles in the isolates appear to depend on the properties of blood cells and the surrounding medium, which may be altered by disease. The isolated nanoparticles, therefore, represent a clinically relevant parameter, even though they may have been created after blood sampling.

\section{Acknowledgments}

The authors are indebted to Maruša Lokar for support in the collaboration between the Laboratory of Clinical Biophysics and FEI Quanta. This work was supported by grants from the Slovenian Research Agency.

\section{Disclosure}

The authors declare there are no competing financial interests to disclose in this work.

\section{References}

1. Wolf $\mathrm{P}$. The nature and significance of platelet products in human plasma. Br J Haematol. 1967;13(3):269-288. 
2. Rumsby MG, Trotter J, Allan D, Michell RH. Recovery of membrane micro-vesicles from human erythrocytes stored for transfusion: a mechanism for the erythrocyte discocyte-to-spherocyte shape transformation. Biochem Soc Trans. 1977;5(1):126-128.

3. Greenwalt TJ. The how and why of exocytic vesicles. Transfusion. 2006;46(1):143-152.

4. Simak J, Gelderman MP. Cell membrane microparticles in blood and blood products: potentially pathogenic agents and diagnostic markers. Transfus Med Rev. 2006;20(1):1-26.

5. Allan D, Billah MM, Finean JB, Michell RH. Release of diacylglycerolenriched vesicles from erythrocytes with increased intracellular $\mathrm{Ca} 2+$. Nature. 1976;261:58-60.

6. Araki T. Release of cholesterol-enriched microvesicles from human erythrocytes caused by hypertonic saline at low-temperatures. FEBS Lett. 1979;97(2):237-240.

7. Hägerstrand H, Isomaa B. Vesiculation induced by amphiphiles in erythrocytes. Biochim Biophys Acta. 1989;982(2):179-186.

8. Hägerstrand H, Bobrowska-Hägerstrand M, Lillsunde I, Isomaa B. Vesiculation induced by amphiphiles and ionophore A23187 in porcine platelets: a transmission electron microscopic study. Chem Biol Interact. 1996;101(2):115-126.

9. Black PH. Shedding from normal and cancer-cell surfaces. New Engl J Med. 1980;303(24):1415-1416.

10. Taylor DD, Chou IN, Black PH. Isolation of plasma-membrane fragments from cultured murine melanoma-cells. Biochem Biophys Res Commun. 1983;113(2):470-476.

11. Kralj-Iglič V, Batista U, Hägerstrand H, Iglič A, Majhenc J, Sok M. On mechanisms of cell plasma membrane vesiculation. Radiol Oncol. 1998;32:119-123.

12. Koga K, Matsumoto K, Akiyoshi T, et al. Purification, characterization and biological significance of tumor-derived exosomes. Anticancer Res. 2005;25(6A):3703-3707.

13. Taylor DD, Black PH. Neoplastic and developmental importance of shed plasma membrane fragments. Am Zool. 1986;26(3): 511-514.

14. Bastida E, Ordinas A, Escolar G, Jamieson GA. Tissue factor in microvesicles shed from U87MG human glioblastoma cells induces coagulation, platelet aggregation, and thrombogenesis. Blood. 1984; 64(1):177-184.

15. Del Conde I, Bharwani LD, Dietzen DJ, Pendurthi U, Thaigarajan P, López JA. Microvesicle-associated tissue factor and Trousseau's syndrome. J Thromb Haemost. 2007;5(1):70-74.

16. Rauch U, Antoniak S. Tissue factor-positive microparticles in blood associated with coagulopathy in cancer. Thromb Haemost. 2007;97(1): 9-10.

17. Tesselaar MET, Romijn FPHTM, van der Linden K, Bertina RM, Osanto S. Microparticle-associated tissue factor activity in cancer patients with and without thrombosis. J Thromb Haemost. 2009;7(8):1421-1423.

18. Junkar I, Šuštar V, Frank M, et al. Blood and synovial microparticles as revealed by atomic force and scanning electron microscope. Open Autoimmun J. 2009;1(9):e50-e58.

19. Mrvar-Brečko A, Šuštar V, Janša V, et al. Isolated microvesicles from peripheral blood and body fluids as observed by scanning electron microscope. Blood Cell Mol Dis. 2010;44(4):307-312.

20. Berckmans RJ, Sturk A, van Tienen LM, Schaap MC, Nieuwland R. Cell-derived vesicles exposing coagulant tissue factor in saliva. Blood. 2011;117(11):3172-3180.

21. Gross PL. Salivary microvesicles clot blood. Blood. 2011;117(11): 2989-2997.

22. Pascual M, Steiger G, Sadallah S, et al. Identification of membrane bound CR1 (CD35) in human urine: evidence for its release by glomerular podocytes. J Exp Med. 1994;179(3):889-899.

23. Ratajczak MZ. Microvesicles: from "dust to crown”. Blood. 2006; 108(9):2885-2886.

24. Diamant M, Tushuizen ME, Sturk A, Nieuwland R. Cellular microparticles: new players in the field of vascular disease? Eur J Clin Invest. 2004;34(6):392-401.
25. Hugel B, Martinez MC, Kunzelmann C, Freyssinet JM. Membrane microparticles: two sides of the coin. Physiology (Bethesda). 2005; 20(1):22-27.

26. Distler JH, Pisetsky DS, Huber LC, Kalden JR, Gay S, Distler O. Microparticles as regulators of inflammation: novel players of cellular crosstalk in the rheumatic diseases. Arthritis Rheum. 2005;52(11): 3337-3348.

27. Ratajczak J, Wysoczynski M, Hayek F, Janowska-Wieczorek A, Ratajczak MZ. Membrane-derived microvesicles: important and underappreciated mediators of cell to cell communication. Leukemia. 2006;20(9):1487-1495.

28. Pisetsky DS. Microparticles as biomarkers in autoimmunity: from dust bin to center stage. Arthritis Res Ther. 2009;11(6):135.

29. Muller I, Klocke A, Alex M, et al. Intravascular tissue factor initiates coagulation via circulating microvesicles and platelets. FASEB J. 2003; 17(3):476-478.

30. Kim HK, Song KS, Park YS, et al. Elevated levels of circulating platelet microparticles, VEGF, IL-6 and RANTES in patients with gastric cancer: possible role of a metastatic predictor. Eur J Cancer. 2003; 39(2):184-191.

31. Kumpers P, Erdbrugger U, Grossheim M, et al. Endothelial microparticles as a diagnostic aid in Churg-Strauss vasculitis-induced cardiomyopathy. Clin Exp Rheumatol. 2008;26(3 Suppl 49):S86-S89.

32. Schetter AJ, Harris CC. Plasma microRNAs: a potential biomarker for colorectal cancer? Gut. 2009;58(10):1318-1319.

33. Piccin A, Murphy WG, Smith OP. Circulating microparticles: pathophysiology and clinical implications. Blood Rev. 2007;21(3):157-171.

34. Sellam J, Proulle V, Jüngel A, et al. Increased levels of circulating microparticles in primary Sjögren's syndrome, systemic lupus erythematosus and rheumatoid arthritis and relation with disease activity. Arthritis Res Ther. 2009;11(5):R156.

35. Orozco AF, Lewis DE. Flow cytometric analysis of circulating microparticles in plasma. Cytometry A. 2010;77(6):502-514.

36. Dey-Hazra E, Hertel B, Kirsch T, et al. Detection of circulating microparticles by flow cytometry: influence of centrifugation, filtration of buffer, and freezing. Vasc Health Risk Manag. 2010;6: 1125-1133.

37. Robert S, Poncelet P, Lacroix R, et al. Standardization of plateletderived microparticle counting using calibrated beads and a Cytomics C500 routine flow cytometer: a first step towards multicenter studies? J Thromb Haemost. 2009;7(1):190-197.

38. Shah MD, Bergeron AL, Dong JF, Lopez JA. Flow cytometric measurement of microparticles: pitfalls and protocol modifications. Platelets. 2008;19(5):365-372.

39. Huica R, Huica S, Moldoveanu E. Flow cytometric assessment of circulating microparticles - towards a more objective analysis. Rom Biotech Lett. 2011;16(3):6271-6277.

40. Diamant M, Nieuwland R, Pablo RF, Sturk A, Smit JW, Radder JK. Elevated numbers of tissue-factor exposing microparticles correlate with components of the metabolic syndrome in uncomplicated type 2 diabetes mellitus. Circulation. 2002;106(19): 2442-2447.

41. Janša R, Šuštar V, Frank M, et al. Number of microvesicles in peripheral blood and ability of plasma to induce adhesion between phospholipid membranes in 19 patients with gastrointestinal diseases. Blood Cells Mol Dis. 2008;41(1):124-132.

42. Yuana Y, Bertina RM, Osanto S. Pre-analytical and analytical issues in the analysis of blood microparticles. Thromb Haemost. 2011;105(3): 396-408.

43. Deuling HJ, Helfrich W. Curvature elasticity of fluid membranes catalog of vesicle shapes. J Phys France. 1976;37(11):1335-1345.

44. Kralj-Iglič V, Babnik B, Gauger DR, May S, Iglič A. Quadrupolar ordering of phospholipid molecules in narrow necks of phospholipid vesicles. J Stat Phys. 2006;125(3):727-752.

45. Iglič A, Babnik B, Gimsa U, Kralj-Iglič V. On the role of membrane anisotropy in beading transition of undulated tubular membrane structures. J Phys A Math Gen. 2005;38:8527-8536. 
46. Šuštar V, Bedina-Zavec A, Štukelj R, et al. Post-prandial rise of microvesicles in peripheral blood of healthy human donors. Lipids Health Dis. 2011;10:47.

47. Pavlič JI, Genova J, Zheliaskova A, Iglič A, Mitov MD. Bending elasticity of lipid membranes in presence of beta 2 glycoprotein I in the surrounding solution. J Phys Conf Ser. 2010;253:012064.

48. Kabaso D, Lokar M, Kralj-Iglič V, Veranic P, Iglic A. Temperature and cholera toxin B are factors that influence formation of membrane nanotubes in RT4 and T24 urothelial cancer cell lines. Int J Nanomedicine. 2011;6:495-509.

49. Kabaso D, Gongadze E, Elter P, et al. Attachment of rod-like (BAR) proteins and membrane shape. Mini Rev Med Chem. 2011;11(4): $272-282$.

50. György B, Módos K, Pállinger E, et al. Detection and isolation of cellderived microparticles are compromised by protein complexes due to shared biophysical parameters. Blood. 2011;117(4):e39-e48.

51. Baj-Krzyworzeka M, Szatanek R, Weglarczyk K, et al. Tumour-derived microvesicles carry several surface determinants and mRNA of tumor cells and transfer some of these determinants to monocytes. Cancer Immunol Immunother. 2006;55(7):808-818.

52. Holmes CE, Levis JE, Ornstein DL. Activated platelets enhance ovarian cancer cell invasion in a cellular model of metastasis. Clin Exp Metab. 2009;26(7):653-661.
53. Janowska-Wieczorek A, Marquez-Curtis LA, Wysoczynski M, Ratajczak MZ. Enhancing effect of platelet-derived microvesicles on the invasive potential of breast cancer cells. Transfusion. 2006;46(7): 1199-1209.

54. Gay LJ, Felding-Habermann B. Contribution of platelets to tumor metastasis. Nat Rev Cancer. 2011;11(2):123-134.

55. Gasic GJ, Gasic TB, Stewart CC. Antimetastatic effects associated with platelet reduction. Proc Natl Acad Sci U SA. 1968;61(1):46-52.

56. Arslan C, Coskun HS. Thrombocytosis in solid tumors: review of the literature. Turk J Haematol. 2005;22:59-64.

57. Prokopi M, Pula G, Mayr U, et al. Proteomic analysis reveals presence of platelet microparticles in endothelial progenitor cell cultures. Blood. 2009;114(3):723-732.

58. Boilard E, Nigrovic PA, Larabee K, et al. Platelets amplify inflammation in arthritis via collagen-dependent microparticle production. Science. 2010;327(5965):580-583.

59. Pap E, Pállinger E, Pásztói M, Falus A. Highlights of a new type of intercellular communication: microvesicle-based information transfer. Inflamm Res. 2009;58(1):1-8.

60. Szajnik M, Czystowska M, Szczepanski MJ, Mandapathil M, Whiteside TL. Tumor-derived microvesicles induce, expand and up-regulate biological activities of human regulatory $\mathrm{T}$ cells (Treg). PLoS One. 2010;5(7):e11469.

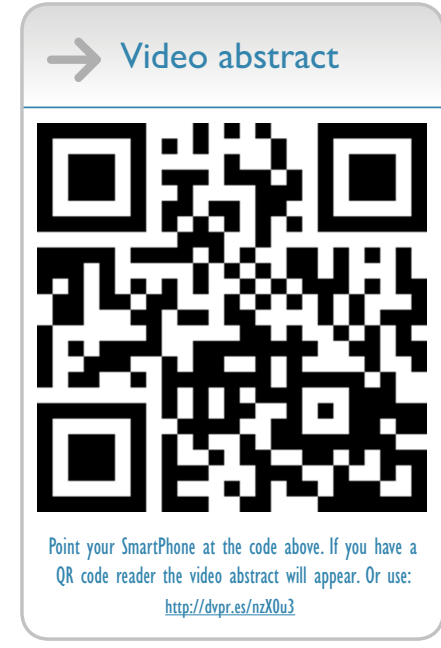

International Journal of Nanomedicine

\section{Publish your work in this journal}

The International Journal of Nanomedicine is an international, peerreviewed journal focusing on the application of nanotechnology in diagnostics, therapeutics, and drug delivery systems throughout the biomedical field. This journal is indexed on PubMed Central, MedLine, CAS, SciSearch ${ }^{\circledR}$, Current Contents ${ }^{\circledR} /$ Clinical Medicine,

\section{Dovepress}

Journal Citation Reports/Science Edition, EMBase, Scopus and the Elsevier Bibliographic databases. The manuscript management system is completely online and includes a very quick and fair peer-review system, which is all easy to use. Visit http://www.dovepress.com/ testimonials.php to read real quotes from published authors. 